\title{
A Group Decision MaKing For CoAstal WETLAND PARK SELECTION APPROACH USING TOPSIS AND INTERVAL-VALUED FUZZY NUMBERS
}

\author{
Chien-Chi Lin ${ }^{1}, *$ and Ming-Ching Lee ${ }^{2}$ \\ ${ }^{1}$ Department of Tourism, Food \& Beverage Management, Chang Jung Christian \\ University, 71101 Tainan, Taiwan \\ ${ }^{2}$ Department of Hospitality Management, Taiwan Shoufu University, 72153 \\ Tainan, Taiwan
}

\begin{abstract}
Due to the increasing awareness of environmental and social issues, sustainable coastal wetland park selection becomes an important problem. The aim of this paper is to develop a new performance evaluation method for multi-attribute decision making (MADM) problems in a group decision environment, based on combining an integrated group Techniques for Order Preferences by Similarity to Ideal Solution (IG-TOPSIS) and interval-valued fuzzy numbers. Coastal wetland park selection often involves uncertain information due to the subjective nature of human judgments. Because human beings are more suitable using linguistic terms rather than crisp values or precise numbers to express what they perceive, the rating values can be expressed in linguistic terms. These linguistic terms, however, are often imprecise or vague. The interval-valued fuzzy sets can provide more flexibility than ordinary fuzzy sets in representing vague or uncertain information. This paper presents an interval-valued fuzzy IG-TOPSIS method, which aims to solve MADM problems in which the preferences of different decision makers are considered and expressed clearly using the concepts of interval-valued fuzzy sets. A case study for evaluating the performances of several sites for coastal wetland park selection is conducted to illustrate the effectiveness of this method.
\end{abstract}

\section{KEYWORDS}

Interval-valued fuzzy sets; TOPSIS; Integrated group TOPSIS; MADM

\section{INTRODUCTION}

Multi-attribute decision making (MADM) is a crucial part of modern decision analysis. It has been widelyapplied in various domains such as economics, sociology,management and engineering science; for example, venture capital project evaluation, economic evaluation, and facility location[1-2]. TOPSIS (technique for order performance by similarity to ideal solution) introduced by Yoon and Hwang [3] is a useful method in solving MADM problems. It helps decision makers organize the problems to be solved, and carry out analysis, comparisons and rankings of the alternatives. TOPSIS is based on the idea that the most favorable alternative should have the shortest distance to an ideal solution [3].

Certain groups constantly make complicated decisions within organizations, so group decision making has drawn increased attention [4, 5, 6, 7]. In extending TOPSIS to a group decision 
making, some works have involved the preference aggregation in the decision making. Shih et al. [8] proposed a post-work to improve TOPSIS as a problem-solving tool. This compensation, however, requires a group decision support system to achieve its purposes. To simplify the decision making activities, Shih et al. [7, 9] further suggested an integrated group TOPSIS (IGTOPSIS) method in which the preferences of decision makers are considered and internally aggregated. IG-TOPSIS can solve MADM problems effectively and is quite effective for realworld applications.

The attribute rating process of MADM problems often involves a complicatedprcedure in which multiple criteria and uncertain conditions must be considered simultaneously. In evaluating the alternatives, qualitative or quantitative assessments are often required to express linguistic terms, which are best represented by fuzzy sets [10-12]. However, the presentation of linguistic terms in the form of ordinary fuzzy sets is not adequate. Interval-valued fuzzy sets, initially proposed by Gorzalczany [13], provide more flexibility to represent vague or uncertain information. Intervalvalued fuzzy sets have been extensivelyused in real-world applications. For example, Liu [1] proposed a weighted aggregation operator based on interval-valued trapezoidal fuzzy numbers. For the MADM problems in which the rating values are generally expressed in linguistic terms, the interval-valued fuzzy numbers can provide more flexibility and can ensure the presentation of linguistic terms as sufficiently clear [10].

In this paper, we develop an interval-valued fuzzy IG-TOPSIS method to solve group decision making problems in which the rating values and weights of attributes are linguistic terms expressed in interval-valued fuzzy numbers. In the next section, this paper briefly introduces the TOPSIS, IG-TOPSIS method, and interval-valued fuzzy sets. Section 3 describes a new method based on the combined concepts of the IG-TOPSIS method and interval-valued fuzzy sets. Section 4 investigates a numerical example, including an application to site selection for a coastal wetland park. Finally, Section 5 draws conclusions.

\section{CONCEPTS OF TOPSIS, IG-TOPSIS, FuZzy SETS, AND INTERVAL- VALUED FUZZY NUMBERS}

\subsection{TOPSIS and IG-TOPSIS Method}

TOPSIS, the famoustypical MADM method, was first developed by Hwang and Yoon [3]. Let $A=\left\{A_{1}, A_{2}, \ldots, A_{m}\right\}$ be a discrete set of $m$ feasible alternatives, $U=\left\{U_{1}, U_{2}, \ldots, U_{m}\right\}$ be a finite set of attributes. The TOPSIS consists of the following steps [14-16]:

Step 1.Normalize the decision matrix.

In general, the attributes ofan MADM problem are benefit attributes and cost attributes. For the purpose of measuring all attributes in dimensionless units and facilitating inter-attribute comparisons, the following formulas are used to normalize each attribute value $x_{i j}$ in decision matrix $X=\left(x_{i j}\right)_{m \times n}$ into a corresponding element $r_{i j}$ in normalized decision matrix given by Eq. (1).

$$
R=\left(r_{i j}\right)_{m x n}=\left[\begin{array}{cccc}
r_{11} & r_{12} & \cdots & r_{1 n} \\
r_{21} & r_{22} & \cdots & r_{2 n} \\
\vdots & \vdots & \vdots & \vdots \\
r_{m 1} & r_{m 1} & \cdots & r_{m n}
\end{array}\right] \text {, }
$$


where

$$
r_{i j}=\frac{x_{i j}}{\sqrt{\sum_{i=1}^{m}\left(x_{i j}\right)^{2}}},
$$

for benefit attribute $x_{i j}$,

and

$$
r_{i j}=1-\frac{x_{i j}}{\sqrt{\sum_{i=1}^{m}\left(x_{i j}\right)^{2}}},
$$

for cost attribute $x_{i j}$.

Step 2. Calculate the weighted normalized decision matrix. Suppose that $W=\left(w_{1}, w_{2}, \ldots, w_{n}\right)^{T}$ is the weight vector of the attributes, where $w_{j} \geq 0, \sum_{j=1}^{n} w_{j}=1$, the weighted normalized decision matrix can be constructed as:

$$
\begin{aligned}
V=\left(w_{j} r_{i j}\right)_{m x n} & =\left(v_{i j}\right)_{m x n} \\
& =\left[\begin{array}{cccc}
v_{11} & v_{12} & \cdots & v_{1 n} \\
v_{21} & v_{22} & \cdots & v_{2 n} \\
\vdots & \vdots & \vdots & \vdots \\
v_{m 1} & v_{m 2} & \cdots & v_{m n}
\end{array}\right] .
\end{aligned}
$$

Step 3.Determine the positive and negative ideal solutions. The positive idealsolution (PIS) $V^{+}$and negative ideal solutions (NIS) $V^{-}$are determined as follows:

$$
\begin{gathered}
V^{+}=\left\{v_{1}^{+}, v_{2}^{+}, \ldots, v_{n}^{+}\right\} \\
V^{-}=\left\{v_{1}^{-}, v_{2}^{-}, \ldots, v_{n}^{-}\right\}
\end{gathered}
$$

where $v_{j}^{+}=\max \left\{v_{i j}\right\}$ and $v_{j}^{-}=\min \left\{v_{i j}\right\}$

Step 4. Measure the distance from positive and negative ideal solutions. The separation of each alternative form the PIS, $S_{i}^{+}$, is given as:

$$
S_{i}^{+}=\left(\sum_{j=1}^{n}\left(v_{i j}-v_{j}^{+}\right)^{2}\right)^{\frac{1}{2}} .
$$

Similarly, the separation form the NIS, $S_{i}^{-}$, is given as:

$$
S_{i}^{-}=\left(\sum_{j=1}^{n}\left(v_{i j}-v_{j}^{-}\right)^{2}\right)^{\frac{1}{2}} \text {. }
$$

Step 5.Calculate the closeness coefficient to the ideal solutions. The closeness coefficient of the $i$ th alternative $A_{i}$ with respect to the ideal solutions is defined as:

$$
C_{i}=\frac{S_{i}^{-}}{S_{i}^{+}+S_{i}^{-}}
$$

Step 6.Rank the preference order.A set of alternatives then can be ranked by preference according to the descending order of $C_{i}$. 


\subsection{IG-TOPSIS Method}

The IG-TOPSIS method was introduced by Shih et al. [7, 9], who considered the separation measures by adopting the arithmetic mean of the individuals for TOPSIS to include the multiple preferences of different decision makers. Compared to the original TOPSIS procedure, IGTOPSIS method offers a general view of TOPSIS with group preference aggregation.

Suppose a MADM problem has $m$ alternatives $A_{1}, A_{2}, \ldots, A_{m}$, and $n$ attributes. Each alternative is evaluated with respect to the $n$ attributes by $K$ decision makers. The procedure of IG-TOPSIS consists of the following steps.

Step 1.Construct a decision matrix $D^{k}, k=1,2, \ldots, k$ for each decision maker. The structure of the decision matrix can be expressed as follows:

$$
D^{k}=\left[\begin{array}{cccc}
x_{11}^{k} & x_{12}^{k} & \cdots & x_{1 n}^{k} \\
x_{21}^{k} & x_{22}^{k} & \cdots & x_{2 n}^{k} \\
\vdots & \vdots & \vdots & \vdots \\
x_{m 1}^{k} & x_{m 2}^{k} & \cdots & x_{m n}^{k}
\end{array}\right]=\left[x_{i j}^{k}\right]_{m \times n},
$$

where $x_{i j}^{k}$ denotes the performance rating of alternative $A_{i}$.

Step 2. Construct the normalized decision matrix $R^{k}=\left[r_{i j}^{k}\right]_{m \times n}$, for each decision maker. The normalized value $r_{i j}^{k}$ can be represented as:

$$
r_{i j}^{k}=\frac{x_{i j}^{k}}{\sqrt{\sum_{j=1}^{n}\left(x_{i j}^{k}\right)^{2}}},
$$

where $i=1, \cdots, m ; j=1, \cdots, n$; and $k=1, \cdots, K$.

Step 3. Construct the normalized weighted decision matrix $V^{k}$. Each decision maker first elicits weights for attributes as $w_{j}^{k}, j=1, \cdots, n$, and $\sum_{j=1}^{n} w_{j}^{k}=1$. For decision makerk, the normalized weighted decision matrix is:

$$
\begin{aligned}
V^{k} & =\left[\begin{array}{cccc}
v_{11}^{k} & v_{12}^{k} & \cdots & v_{1 n}^{k} \\
v_{21}^{k} & v_{22}^{k} & \cdots & v_{2 n}^{k} \\
\vdots & \vdots & \vdots & \vdots \\
v_{m 1}^{k} & v_{m 2}^{k} & \cdots & v_{m n}^{k}
\end{array}\right] \\
& =\left[\begin{array}{cccc}
w_{1}^{k} v_{11}^{k} & w_{2}^{k} v_{12}^{k} & \cdots & w_{n}^{k} v_{1 n}^{k} \\
w_{1}^{k} v_{21}^{k} & w_{2}^{k} v_{22}^{k} & \cdots & w_{n}^{k} v_{2 n}^{k} \\
\vdots & \vdots & \vdots & \vdots \\
w_{1}^{k} v_{m 1}^{k} & w_{2}^{k} v_{m 2}^{k} & \cdots & w_{n}^{k} v_{m n}^{k}
\end{array}\right]
\end{aligned}
$$


Step 4. Determine both PIS $V^{k+}$ and NIS $V^{k-}$. For each decision maker, his or her $V^{k+}$ and $V^{k-}$ are:

$$
\begin{aligned}
V^{k+} & =\left\{v_{1}^{k+}, \cdots, v_{n}^{k+}\right\} \\
& =\left\{\left(\max _{i} v_{i j}^{k} \mid j \in J\right),\left(\min _{i} r_{i j}^{k} \mid j \in J^{\prime}\right)\right\}, \\
V^{k-} & =\left\{v_{1}^{k-}, \cdots, v_{n}^{k-}\right\} \\
& =\left\{\left(\min _{i} v_{i j}^{k} \mid j \in J\right),\left(\max _{i} r_{i j}^{k} \mid j \in J^{\prime}\right)\right\},
\end{aligned}
$$

where $J$ is associated with the benefit criteria and $J^{\prime}$ is associated with the cost criteria.

Step 5.Calculate the separation measure $S^{k+}$ and $S^{k-}$ from PIS and NIS for each decision maker. For decision makerk, the individual separation measures of each alternative from PIS and NIS are:

$$
\begin{aligned}
& S_{i}^{k+}=\left[\sum_{j=1}^{n}\left(v_{i j}^{k}-v_{i j}^{k+}\right)^{2}\right]^{1 / 2}, i=1, \ldots m ; \\
& S_{i}^{k-}=\left[\sum_{j=1}^{n}\left(v_{i j}^{k}-v_{i j}^{k-}\right)^{2}\right]^{1 / 2}, i=1, \ldots m .
\end{aligned}
$$

Step 6. Calculate the group separation measures using the arithmetic mean. The two group separation measures are:

$$
\begin{aligned}
& \bar{S}_{i}^{+}=\frac{1}{K}\left(\sum_{k=1}^{K} S_{i}^{k+}\right), \\
& \bar{S}_{i}^{-}=\frac{1}{K}\left(\sum_{k=1}^{K} S_{i}^{k-}\right) .
\end{aligned}
$$

Step 7. Calculate the relative closeness $\bar{C}_{i}^{*}$ to the ideal solution for the group. $\bar{C}_{i}^{*}$ can be defined as:

$$
\bar{C}^{*}=\frac{\bar{S}_{i}^{-}}{\bar{S}_{i}^{+}+\bar{S}_{i}^{-}}, \quad i=1, \ldots, m .
$$

Step 8.Rank the order. According to the relative closeness to the ideal solution, the greater the $\bar{C}_{i}^{*}$ is, the more favorable the alternative $A_{i}$ is.

\subsection{Fuzzy sets and Interval-Valued Fuzzy Numbers}

The rating values in MADM problems are usually expressed in linguistic terms. Because these linguistic terms are often vague or imprecise, it may be moreappropriate to treat them as fuzzy rather than precise. A fuzzy set is a collection of elements in a universe. Each fuzzy set is specified by a membership function, which assigns to a value within the unit interval $[0,1]$. The assigned value is called the degree of membership. The element totally belongs to the set if the assigned value is 1 . The given element does not belong to the set if the assigned value is 0 . The element only partially belongs to the set if the value lies within the interval $[0,1]$. Consequently, any fuzzy set can be uniquely determined by its membership function [17].

Triangular fuzzy number is a widely used type of fuzzy set since it can be easily handled arithmetically and interpreted intuitively. A triangular fuzzy number $A_{T}$, denoted as $A_{T}=(a, b, c)$, where $a \leq b \leq c$, has the triangular-shape membership function [18-19]: 


$$
\begin{aligned}
& \mu_{A_{T}}(x)= \\
& \left\{\begin{array}{l}
(x-a) /(b-a), a \leq x \leq b, \\
(c-x) /(c-b), b \leq x \leq c, \\
0, \text { otherwise. }
\end{array}\right.
\end{aligned}
$$

where $b$ denotes the element with the largest membership value, $a$ and $c$ denote the lower and upper values, respectively.

Let two positive triangular fuzzy numbers, $\tilde{A}$ and $\widetilde{B}$, be $\left(a_{1}, b_{1}, c_{1}\right)$ and $\left(a_{2}, b_{2}, c_{2}\right)$, respectively. Some arithmetic operations of $\tilde{A}$ and $\widetilde{B}$ can be defined as follows [18-19]:

$$
\begin{aligned}
& \tilde{A}(+) \tilde{B}=\left(a_{1}+a_{2}, b_{1}+b_{2}, c_{1}+c_{2}\right), \\
& \tilde{A}(-) \tilde{B}=\left(a_{1}-c_{2}, b_{1}-b_{2}, c_{1}-a_{2}\right), \\
& \tilde{A}(\cdot) \tilde{B}=\left(a_{1} \cdot a_{2}, b_{1} \cdot b_{2}, c_{1} \cdot c_{2}\right), \\
& \tilde{A}(\div) \tilde{B}=\left(a_{1} / c_{2}, b_{1} / b_{2}, c_{1} / a_{2}\right), \\
& k>0, k \cdot \tilde{A}=\left(k a_{1}, k b_{1}, k c_{1}\right) .
\end{aligned}
$$

However, linguistic terms in the form of ordinary fuzzy sets are not adequately clear [10, 20-21]. Gorzalczany [8] proposed the concept of interval-valued fuzzy sets which can provide more flexibility than ordinary fuzzy sets and can ensure that the presentation of linguistic terms is sufficiently clear.

An interval-valued fuzzy set $A$ defined on $(-\infty,+\infty)$ is given by:

$$
\begin{aligned}
& A=\left\{x,\left[\mu_{\mathrm{A}}^{\mathrm{L}}(x), \mu_{\mathrm{A}}^{\mathrm{U}}(x)\right]\right\} \\
& \mu_{\mathrm{A}}^{\mathrm{L}}(x), \mu_{\mathrm{A}}^{\mathrm{U}}(x): X \rightarrow[0,1] \quad \forall x \in X, \mu_{A}^{L} \leq \mu_{A}^{U}
\end{aligned}
$$

where $\mu_{A}^{L}(x)$ is the lower limit of degree of membership and $\mu_{A}^{U}(x)$ is the upper limit of degree of membership. Figure 1 illustrates the membership value at $x$ ' of interval-valued fuzzy set $A$. Thereby, the minimum and maximum membership value of $x^{\prime}$ are $\mu_{A}^{L}(x)$ and $\mu_{A}^{U}(x)$, respectively.

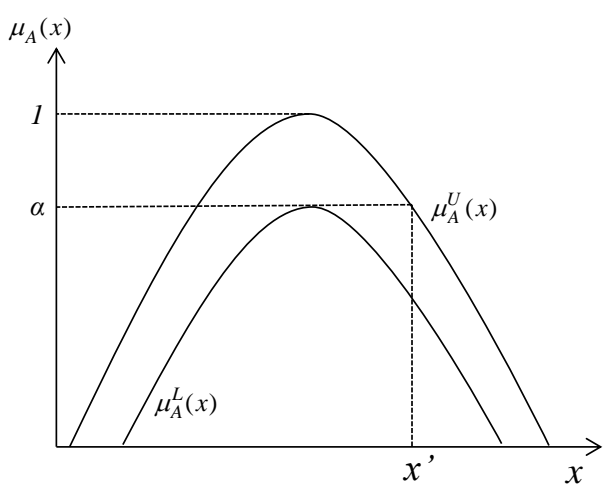

Figure 1.Interval-valued fuzzy set. 
Two triangular interval-valuedfuzzy numbers can be represented as: $A=\left[\left(a_{1}, a_{1}^{\prime}\right), a_{2},\left(a_{3}, a_{3}^{\prime}\right)\right]$ and $B=\left[\left(b_{1}, b_{1}^{\prime}\right), b_{2},\left(b_{3}, b_{3}^{\prime}\right)\right]$, respectively. The arithmetic operations between $A$ and $B$ are proposed by $[10,22]$ as follows:

(1) Addition of interval-valued fuzzy numbers $\oplus$ :

$$
\begin{aligned}
A \oplus B & =\left[\left(a_{1}, a_{1}^{\prime}\right), a_{2},\left(a_{3}, a_{3}^{\prime}\right)\right] \oplus\left[\left(b_{1}, b_{1}^{\prime}\right), b_{2},\left(b_{3}, b_{3}^{\prime}\right)\right] \\
& =\left[\left(a_{1}+b_{1}, a_{1}^{\prime}+b_{1}^{\prime}\right), a_{2}+b_{2},\left(a_{3}+b_{3}, a_{3}^{\prime}+b_{3}^{\prime}\right)\right] .
\end{aligned}
$$

(2) Subtraction of interval-valued fuzzy numbers $\Theta$ :

$$
\begin{aligned}
A \Theta B & =\left[\left(a_{1}, a_{1}^{\prime}\right), a_{2},\left(a_{3}, a_{3}^{\prime}\right)\right] \Theta\left[\left(b_{1}, b_{1}^{\prime}\right), b_{2},\left(b_{3}, b_{3}^{\prime}\right)\right] \\
& =\left[\left(a_{1}-b_{3}^{\prime}, a_{1}^{\prime}-b_{3}\right), a_{2}-b_{2},\left(a_{3}-b_{1}^{\prime}, a_{3}^{\prime}-b_{1}\right)\right] .
\end{aligned}
$$

(3) Multiplicationof interval-valued fuzzy numbers $\otimes$ :

$$
\begin{aligned}
A \otimes B & =\left[\left(a_{1}, a_{1}^{\prime}\right), a_{2},\left(a_{3}, a_{3}^{\prime}\right)\right] \otimes\left[\left(b_{1}, b_{1}^{\prime}\right), b_{2},\left(b_{3}, b_{3}^{\prime}\right)\right] \\
& =\left[\left(a_{1} \times b_{1}, a_{1}^{\prime} \times b_{1}^{\prime}\right), a_{2} \times b_{2},\left(a_{3} \times b_{3}, a_{3}^{\prime} \times b_{3}^{\prime}\right)\right] .
\end{aligned}
$$

(4) Generalized divisionof interval-valued fuzzy numbers $\div$ :

$$
\begin{aligned}
A \otimes B & =\left[\left(a_{1}, a_{1}^{\prime}\right), a_{2},\left(a_{3}, a_{3}^{\prime}\right)\right] \otimes\left[\left(b_{1}, b_{1}^{\prime}\right), b_{2},\left(b_{3}, b_{3}^{\prime}\right)\right] \\
& =\left[\left(a_{1} \times b_{1}, a_{1}^{\prime} \times b_{1}^{\prime}\right), a_{2} \times b_{2},\left(a_{3} \times b_{3}, a_{3}^{\prime} \times b_{3}^{\prime}\right)\right] .
\end{aligned}
$$

The distance between $\tilde{A}$ and $\widetilde{B}$ is [23]:

$$
\begin{aligned}
d(A, B)= & \sum_{i=1}^{n}\left(\max \left(\left|a_{1}^{\prime}-b_{1}^{\prime}\right|,\left|a_{1}-b_{1}\right|\right)\right. \\
& \left.+\left|a_{2}-b_{2}\right|+\max \left(\left|a_{3}^{\prime}-b_{3}^{\prime}{ }_{3}\right|,\left|a_{3}-b_{3}\right|\right)\right) .
\end{aligned}
$$

\section{The Proposed InTerval - VAlued Fuzzy IG-Topsis Method}

Let $X^{k}=\left[x_{i j}^{k}\right]_{n \times m}$ be a fuzzy decision matrix for a MADM problem in which $A_{1}, A_{2}, \ldots, A_{m}$ are malternatives and $U_{1}, U_{2}, \ldots, U_{m}$ are $n$ attributes. Eachalternative is evaluated with respect to the attributes by $K$ decision makers. The evaluated performance of alternative $A_{i}$ with respect to attribute $U_{i}$ isdenoted as $x_{i j}$. As illustrated in Figure. 2, $x_{i j}$ is expressed in triangular intervalvalued fuzzy numbers. The $x$ can be demonstrated as $x=\left[\left(a, a^{\prime}\right), b,\left(c, c^{\prime}\right)\right]$. 


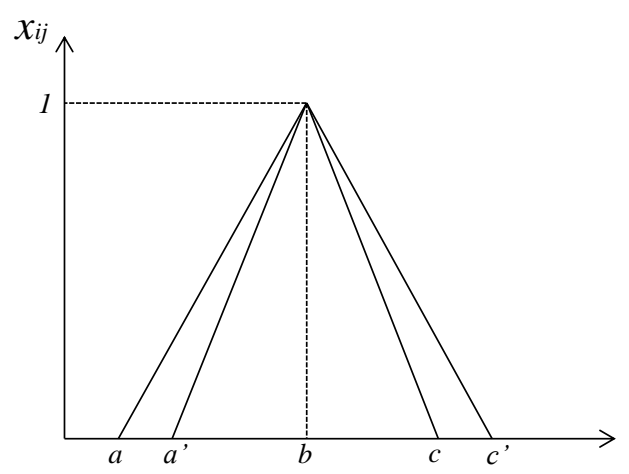

Figure 2. Interval-valued triangular fuzzy number.

The proposed method can be defined as follows.

Step 1.Construct a decision matrix $D^{k}=\left[x_{i j}^{k}\right]_{n x m}$ and normalized decision matrix $R_{k}$. Given $x_{i j}^{k}=\left[\left(a_{i j}^{k}, a_{i j}^{\prime k}\right), b_{i j}^{k},\left(c_{i j}^{\prime k}, c_{i j}^{k}\right)\right]$, the normalized decision matrix $R_{k}$ can be calculated as [22, 5]:

$$
\begin{gathered}
r_{i j}^{k}=\left[\left(\frac{a_{i j}^{k}}{c_{j}^{+k}}, \frac{a_{i j}^{\prime k}}{c_{j}^{+k}}\right), \frac{b_{i j}^{k}}{c_{j}^{+k}},\left(\frac{c_{i j}^{\prime k}}{c_{j}^{+k}}, \frac{c_{i j}^{k}}{c_{j}^{+k}}\right)\right], j \in J, \\
r_{i j}^{k}=\left[\left(\frac{a_{i j}^{-k}}{a_{i j}^{\prime k}}, \frac{a_{i j}^{-k}}{a_{i j}^{k}}\right), \frac{a_{i j}^{-k}}{b_{i j}^{k}},\left(\frac{a_{i j}^{-k}}{c_{i j}^{k}}, \frac{a_{i j}^{-k}}{c_{i j}^{\prime k}}\right)\right], j \in J^{\prime}, \\
c_{j}^{+k}=\max \left(c_{i j}^{k}\right), \text { if } j \in J, \\
a_{j}^{-k}=\min \left(a_{i j}^{\prime k}\right), \text { if } j \in J^{\prime} .
\end{gathered}
$$

where $J$ is associated with the benefit criteria and $J^{\prime}$ is associated with the cost criteria.

Step 2. Construct the normalized weighted decision matrix $V^{k}$. By considering the different importance weights of each attribute, we can construct the weighted normalized fuzzy decision matrix for decision maker $k$ as $V=\left[v_{i j}^{k}\right]_{n \times m}$. According to Eq. (21), the multiply operator can be applied as:

$$
\begin{aligned}
v_{i j}^{k} & =v_{i j}^{k} \times w_{j}^{k} \\
& =\left(\left(r_{1 i j}^{k} \times w_{1 j}^{k}, r_{1 i j}^{\prime k} \times w_{1 j}^{\prime k}\right), r_{2 i j}^{k} \times w_{2 j}^{k},\left(r_{3 i j}^{k} \times w_{3 j}^{k}, r_{3 i j}^{\prime k} \times w_{3 j}^{\prime k}\right)\right) \\
& =\left(\left(e_{i j}^{k}, e_{i j}^{\prime k}\right), f_{i j}^{k},\left(g_{i j}^{k}, g_{i j}^{\prime k}\right)\right)
\end{aligned}
$$

Step 3. Determine positive ideal and negative ideal solution $v^{k+}$ and $v^{k-}$ as:

$$
\begin{aligned}
V^{k+} & =\left\{\left(\max _{i} r_{i j}^{k} \mid j \in J\right) \text { or }\left(\min _{i} r_{i j}^{k} \mid j \in J^{\prime}\right)\right\} \\
& =\left[\left(\alpha_{j}^{k+}, \alpha_{j}^{k+}\right), \beta_{j}^{k+},\left(\gamma_{j}^{k+}, \gamma_{j}^{\prime k+}\right)\right], \quad j=1, \ldots, n \\
V^{k-} & =\left\{\left(\min _{i} r_{i j}^{k} \mid j \in J\right) \text { or }\left(\max _{i} r_{i j}^{k} \mid j \in J^{\prime}\right)\right\} \\
& =\left[\left(\alpha_{j}^{k-}, \alpha_{j}^{k-}\right), \beta_{j}^{k-},\left(\gamma_{j}^{k-}, \gamma_{j}^{\prime k-}\right)\right], \quad j=1, \ldots, n
\end{aligned}
$$

where $J$ is associated with the benefit criteria and $J^{\prime}$ is associated with the cost criteria. 
Step 4. Calculate the separation measures from PISand NIS individually according to Eq. (23).For decision maker $k$, his or her separation measures from PIS and NIS are:

$$
\begin{gathered}
S_{i}^{k+}=\sum_{j}^{n}\left(\max \left(\left|e_{i j}^{\prime k}-\alpha_{j}^{\prime k+}\right|,\left|e_{i j}^{k}-\alpha_{j}^{k+}\right|\right)+\left|f_{i j}^{k}-\beta_{j}^{k+}\right|\right. \\
\left.\quad+\max \left(\left|g_{i j}^{\prime k}-\gamma_{j}^{\prime k+}\right|,\left|g_{i j}^{k}-\gamma_{j}^{k+}\right|\right)\right) \\
S_{i}^{k-}=\sum_{j}^{n}\left(\max \left(\left|e_{i j}^{\prime k}-\alpha_{j}^{\prime k-}\right|,\left|e_{i j}^{k}-\alpha_{j}^{k-}\right|\right)+\left|f_{i j}^{k}-\beta_{j}^{k-}\right|\right. \\
\left.+\max \left(\left|g_{i j}^{\prime k}-\gamma_{j}^{\prime k-}\right|,\left|g_{i j}^{k}-\gamma_{j}^{k-}\right|\right)\right)
\end{gathered}
$$

Step 5. Calculate the group separation measures using the arithmetic mean. The two group separation measures are:

$$
\begin{aligned}
& \bar{S}_{i}^{+}=\frac{1}{K}\left(\sum_{k=1}^{K} S_{i}^{k+}\right) \\
& \bar{S}_{i}^{-}=\frac{1}{K}\left(\sum_{k=1}^{K} S_{i}^{k-}\right) .
\end{aligned}
$$

Step 6. Calculate the relative closeness $\bar{C}_{i}^{*}$ to the ideal solution for the group. $\bar{C}_{i}^{*}$ can be defined as:

$$
\bar{C}^{*}=\frac{\bar{S}_{i}^{-}}{\bar{S}_{i}^{+}+\bar{S}_{i}^{-}}, \quad i=1, \ldots, m .
$$

Step 7. Rank the order. According to the relative closeness to the/ ideal solution, the greater the $\bar{C}_{i}^{*}$ is, the more favorable the alternative $A_{i}$ is.

\section{A Casestudy For Site Selection Of Coastal Wetland Parks}

In this section, to illustrate how the present fuzzy MADMmethod works, we present a case study that involves the site selection of natural-based parks on coastal wetlands.

Taiwan is surrounded by the sea. The formation of many coastal wetlands is due to the natural features. Many developed countries have actively promoted nature-based parks on coastal wetlands to protect natural resources and promote environmental education. Based on a literature review of the evaluation of site selections of natural-base resort parks, this study constructed five attributes for site selection. The attributesare:

1)sightseeing attractions $(\mathrm{C} 1)$;

2) transportation convenience $(\mathrm{C} 2)$;

3) physico-ecological carrying capacity (C3);

4) local resident attitudes (C4); and

5) peripheral attractions (C5).

The three alternatives are Dadu coastal wetlands (A1), Fangyuan coastal wetlands (A2), and Dacheng coastal wetlands (A3).

The relative importance weights of the five attributes are described using linguistic terms, such as "low","medium", and "high", which are defined in Table 1. The ratings are also characterized by 
linguistic terms suchas "poor", "fair", and "good", which are defined in Table 2. Thethree decision makers express their opinions on the importance weights ofthe five attributes and the ratings of each alternative with respect tothe five attributes independently. Tables 3 and 4 show the originalassessment information provided by the three decision makers. The weighted normalized fuzzy decision matrixfor each decision maker is calculated using Eq. (25). Table 5 shows the weighted normalized fuzzy decision matrix of the first decision maker. Individual separation measures and group separations are calculated using Eqs. (27), (28) and (29). Applying Eq. (30), the relative closeness for the group is obtained as follows.

$$
\begin{aligned}
& \bar{C}_{1}^{*}=0.43 ; \\
& \bar{C}_{2}^{*}=0.69 ; \\
& \bar{C}_{3}^{*}=0.47 .
\end{aligned}
$$

Therefore, the ranking of the three alternatives is generated as the follows:

$$
A_{2} \succ A_{3} \succ A_{1}
$$

Obviously, the best selection is the Fangyuan coastal wetlands.

Table 1. Definitions of linguistic terms for the importance of each attribute.

\begin{tabular}{ll}
\hline Linguistic terms & Interval-valued fuzzy number \\
\hline Very low $(\mathrm{VL})$ & {$[(0,0) ; 0 ;(0.1,0.15)]$} \\
Low $(\mathrm{L})[(0,0.05)$ & {$[(0,0.05) ; 0.1 ;(0.25,0.35)]$} \\
Medium low (ML) & {$[(0,0.15) ; 0.3 ;(0.45,0.55)]$} \\
Medium (M) & {$[(0.25,0.35), 0.5,(0.65,0.75)]$} \\
Medium high (MH) & {$[(0.45,0.55), 0.7,(0.8,0.95)]$} \\
High $(\mathrm{H})$ & {$[(0.55,0.75), 0.9,(0.95,1)]$} \\
Very high $(\mathrm{VH})$ & {$[(0.85,0.95), 1,(1,1)]$} \\
\hline
\end{tabular}

Table 2.Definitions of linguistic terms for the rating values.

\begin{tabular}{ll}
\hline Linguistic terms & $\begin{array}{l}\text { Interval-valued fuzzy } \\
\text { number }\end{array}$ \\
\hline Very Poor (VP) & {$[(0,0) ; 0 ;(1,1.5)]$} \\
Poor (P) & {$[(0,0.5) ; 1 ;(2.5,3.5)]$} \\
Moderately Poor (MP) & {$[(0,1.5) ; 3 ;(4.5,5.5)]$} \\
Fair (F) & {$[(2.5,3.5), 5,(6.5,7.5)]$} \\
Moderately Good (MG) & {$[(4.5,5.5), 7,(8,9.5)]$} \\
Good (G) & {$[(5.5,7.5), 9,(9.5,10)]$} \\
Very Good(VG) & {$[(8.5,9.5), 10,(10,10)]$} \\
\hline
\end{tabular}

Table3.The importance of each attribute.

\begin{tabular}{llll}
\hline Attributes & $\begin{array}{l}\text { Decision } \\
\text { Maker 1 }\end{array}$ & $\begin{array}{l}\text { Decision } \\
\text { Maker 2 }\end{array}$ & $\begin{array}{l}\text { Decision } \\
\text { Maker 3 }\end{array}$ \\
\hline $\mathrm{C}_{1}$ & $\mathrm{VH}$ & $\mathrm{VH}$ & $\mathrm{VH}$ \\
$\mathrm{C}_{2}$ & $\mathrm{H}$ & $\mathrm{H}$ & $\mathrm{MH}$ \\
$\mathrm{C}_{3}$ & $\mathrm{VH}$ & $\mathrm{VH}$ & $\mathrm{MH}$ \\
$\mathrm{C}_{4}$ & $\mathrm{H}$ & $\mathrm{MH}$ & $\mathrm{VH}$ \\
$\mathrm{C}_{5}$ & $\mathrm{M}$ & $\mathrm{MH}$ & $\mathrm{M}$ \\
\hline
\end{tabular}


Table4.Decision makers' assessments based on each attribute.

\begin{tabular}{|c|c|c|c|c|}
\hline Attributes & Alternatives & $\begin{array}{l}\text { Decision } \\
\text { Maker } 1\end{array}$ & $\begin{array}{l}\text { Decision } \\
\text { Maker } 2 \\
\end{array}$ & $\begin{array}{l}\text { Decision } \\
\text { Maker } 3 \\
\end{array}$ \\
\hline \multirow[t]{3}{*}{$\mathrm{C}_{1}$} & $\mathrm{~A}_{1}$ & MG & MG & G \\
\hline & $\mathrm{A}_{2}$ & VG & G & G \\
\hline & $\mathrm{A}_{3}$ & G & MG & MG \\
\hline \multirow[t]{3}{*}{$\mathrm{C}_{2}$} & $\mathrm{~A}_{1}$ & $\mathrm{~F}$ & MP & $\mathrm{F}$ \\
\hline & $\mathrm{A}_{2}$ & $\mathrm{~F}$ & MP & $\mathrm{F}$ \\
\hline & $\mathrm{A}_{3}$ & MP & F & F \\
\hline \multirow[t]{3}{*}{$\mathrm{C}_{3}$} & $\mathrm{~A}_{1}$ & MG & G & G \\
\hline & $\mathrm{A}_{2}$ & MG & G & MG \\
\hline & $\mathrm{A}_{3}$ & MG & G & MG \\
\hline \multirow[t]{3}{*}{$\mathrm{C}_{4}$} & $\mathrm{~A}_{1}$ & $\mathrm{~F}$ & MG & $\mathrm{F}$ \\
\hline & $\mathrm{A}_{2}$ & MG & MG & $\mathrm{F}$ \\
\hline & $\mathrm{A}_{3}$ & F & MG & MP \\
\hline \multirow[t]{3}{*}{$\mathrm{C}_{5}$} & $\mathrm{~A}_{1}$ & MG & G & G \\
\hline & $\mathrm{A}_{2}$ & G & VG & G \\
\hline & $\mathrm{A}_{3}$ & MP & F & MP \\
\hline
\end{tabular}

Table5.The weighted normalized fuzzy decision matrix for the first decision maker.

\begin{tabular}{llllll}
\hline & C1 & C2 & C3 & C4 & C5 \\
\hline \multirow{2}{*}{ A1 } & {$[(0.516,0.67), 0.7$} & {$[(0.10,0.26), 0.3$} & {$[(0.56,0.72), 0.8$} & {$[(0.29,0.45), 0.58$} & {$[(0.58,0.75), 0.8$} \\
& $9,(0.84,0.91)]$ & $8,(0.43,0.50)]$ & $7,(0.91,0.97)]$ & $,(0.63,0.71)]$ & $6,(0.91,0.98)]$ \\
\hline \multirow{2}{*}{ A2 } & {$[(0.66,0.80), 0.93$} & {$[(0.14,0.32), 0.4$} & {$[(0.44,0.61), 0.7$} & {$[(0.39,0.55), 0.68$} & {$[(0.62,0.81), 0.9$} \\
& $,(0.99,1.00)]$ & $4,(0.50,0.57)]$ & $3,(0.78,0.85)]$ & $,(0.74,0.83)]$ & $3,(1.00,1.00)]$ \\
\hline \multirow{2}{*}{ A3 } & {$[(0.45,0.67), 0.74$} & {$[(0.21,0.35), 0.4$} & {$[(0.52,0.66), 0.7$} & {$[(0.19,0.26), 0.39$} & {$[(0.12,0.14), 0.2$} \\
& $,(0.79,0.86)]$ & $7,(0.54,0.62)]$ & $9,(0.84,0.88)]$ &,$(0.46,0.55]$ & $7,(0.34,0.42)]$ \\
\hline
\end{tabular}

\section{CONClusions}

MADM has been widely appliedin solving real world decision making problems. Most MADM problems include quantitative or qualitative attributes, which are often assessed using linguistic terms. Interval-valued fuzzy set is effective for contending with such decision problems. This paper presents a novel method incorporating the concepts of IG-TOPSIS and interval-valued fuzzy numbers to solve MADM problems. This study considered and expressed clearly the preferences of different decision makers using the concepts of interval-valued fuzzy sets. A case study involving the evaluation of the performance of three sites for coastal wetland park selections was conducted to examine the applicability of the proposed method. This proposed method is easy to understand and quite effective for real-world applications.

\section{REFERENCES}

[1] Liu, P. A weighted aggregation operators multi-attribute group decision-making method based on interval-valued trapezoidal fuzzy numbers. Expert Systems with Applications2011, 38(1), 1053-1060.

[2] Prato, T. Multiple attribute decision analysis for ecosystem management. Ecological Economics1999, 30(2), 207-222.

[3] Yoon, K., Hwang, C. L. Manufacturing plant location analysis by multiple attribute decision making: Part I - single-plant strategy. International Journal of Production Research1985, 23, 345-359.

[4] Chu, T. C. Facility location selection using fuzzy TOPSIS under group decision, International Journal of Uncertainty. Fuzziness and Knowledge-Based Systems2002, 10(6), 687-701. 
[5] Chen, C.-T. Extensions of the TOPSIS for group decision-making under fuzzy environment. Fuzzy Sets and Systems2000, 114(1), 1-9.

[6] Parkan, C., Wu, M. L. Process selection with multiple objective and subjective attributes. Production Planning and Control1998, 9(2), 189-200.

[7] Shih, H.-S., Shyur, H.-J., Lee, E. S. An extension of TOPSIS for group decision making. Mathematical and Computer Modelling2007, 45(7-8), 801-813.

[8] Shih, H. S., Wang, C. H., Lee, E. S. A multi-attribute GDSS for aiding problem-solving. Mathematical and Computer Modelling2004, 39(11), 1397-1412.

[9] Shih, H.-S. Incremental analysis for MCDM with an application to group TOPSIS. European Journal of Operational Research2008, 186(2), 720-734.

[10] Kuo, M.-S., Liang, G.-S. A soft computing method of performance evaluation with MCDM based on interval-valued fuzzy numbers. Applied Soft Computing2012, 12(1), 476-485.

[11] Carlsson, C., Fullér, R. Fuzzy multiple criteria decision making: Recent developments. Fuzzy Sets and Systems 1996, 78(2), 139-153.

[12] Triantaphyllou, E., Lin, C.-T. Development and evaluation of five fuzzy multiattribute decisionmaking methods. International Journal of Approximate Reasoning1996, 14(4), 281-310.

[13] Gorzałczany, M. B. A method of inference in approximate reasoning based on interval-valued fuzzy sets. Fuzzy Sets and Systems1987, 21(1), 1-17.

[14] Yue, Z. A method for group decision-making based on determining weights of decision makers using TOPSIS. Applied Mathematical Modelling2011, 35(4), 1926-1936.

[15] Jahanshahloo, G. R., Lotfi, F. H., Izadikhah, M. An algorithmic method to extend TOPSIS for decision-making problems with interval data. Applied Mathematics and Computation2006, 175(2), 1375-1384.

[16] Chamodrakas, I., Leftheriotis, I., Martakos, D. In-depth analysis and simulation study of an innovative fuzzy approach for ranking alternatives in multiple attribute decision making problems based on TOPSIS. Applied Soft Computing2011, 11(1), 900-907.

[17] Mokhtarian, M. N. A new fuzzy weighted average (FWA) method based on left and right scores: An application for determining a suitable location for a gas oil station. Computers \& Mathematics with Applications2011, 61(10), 3136-3145.

[18] Liu, H.-T., Wang, C.-H. An advanced quality function deployment model using fuzzy analytic network process. Applied Mathematical Modelling2010, 34(11), 3333-3351.

[19] Chang, D.-Y. Applications of the extent analysis method on fuzzy AHP. European Journal of Operational Research1996, 95(3), 649-655.

[20] Bigand, A., Colot, O. Fuzzy filter based on interval-valued fuzzy sets for image filtering. Fuzzy Sets and Systems2010, 161(1), 96-117.

[21] Karnik, N., Mendel, J. Operations on type-2 fuzzy sets. Fuzzy Sets and Systems2001, 122(2), 327348.

[22] Ashtiani, B., Haghighirad, F., Makui, A., Montazer, G. a. Extension of fuzzy TOPSIS method based on interval-valued fuzzy sets. Applied Soft Computing2009, 9(2), 457-461.

[23] Grzegorzewski, P. Distances between intuitionistic fuzzy sets and/or interval-valued fuzzy sets based on the Hausdorff metric. Fuzzy Sets and Systems2004, 148(2), 319-328. 